\title{
Hiperdocumento Didático sobre o Sistema Brasileiro de Classificação de Solos *
}

\author{
Edilson Pontarolo \\ Leila Maria Araújo Santos \\ Luís Fernando Maximo \\ José Valdeni de Lima \\ Rosa Maria Vicari
}

\begin{abstract}
Resumo: O acesso linear ao grande volume de informações sobre o Sistema Brasileiro de Classificação de Solos afeta negativamente a motivação dos alunos de ciência do solo. Este trabalho descreve a modelagem de uma aplicação computacional organizada para capturar uma abordagem didática do tema. A solução modelada consiste de um hiperdocumento que agrega informações estruturadas e conteúdo hipermídia (texto, hipertexto, imagens, áudio e vídeo) sobre o processo de classificação de solos. Um protótipo da aplicação foi implementado e disponibilizado na Internet, baseado inteiramente em ferramentas de software de acesso gratuito. Entretanto, sua validação por alunos de ciência do solo não foi possível até o momento.
\end{abstract}

Palavras-chave: classificação de solos, multimídia educacional, educação a distância.

\section{An Educational Hyperdocument on the Brazilian Soil Taxonomy}

Abstract: The linear access to the huge amount of information on the Brazilian Soil Taxonomy affects negatively the motivation of soil science students. This paper describes the modeling of a computational application organized in order to capture an educational approach to the theme. The modeled solution consists of a hyperdocument that aggregates structured information and hypermedia content (text, hypertext, images, audio and video) on soil classification process. An application prototype was implemented and made available on the Internet, totally based on free software tools. However, its validation by soil science students was not possible to this moment.

Keywords: soil classification, educational multimedia, distance learning.

Uma versão preliminar deste projeto foi apresentada à disciplina "Hiperdocumentos como Material Didático" do PPGIE UFRGS no segundo semestre de 2004.

** Professor do CEFET-PR Pato Branco, Mestre em Engenharia Elétrica e Informática Industrial, Doutorando do PPGIE UFRGS, edi@pb.cefetpr.br, http://iate.ufrgs.br/ edilson.

Professora da ULBRA Santa Maria, Mestre em Educação, Doutoranda do PPGIE UFRGS, leilamas@terra.com.br.

.... Professor da UNIVALI, Mestre em Educação, Doutorando do PPGIE UFRGS, fernandomax@univali.br.

....* Professor do Instituto de Informática e do PPGIE UFRGS, Doutor em Informática, valdeni@inf.ufrgs.br.

Professora do Instituto de Informática e do PPGIE UFRGS, Doutora em Informática, rosa@inf.ufrgs.br, http://www.inf.ufrgs.br/ rosa. 


\section{$1 \quad$ As dificuldades no ensino de classificação de solos}

A adequada classificação de um solo permite estabelecer correlações com sua gênese e evolução, assim como com fatores ambientais e econômicos relativos a sua ocupação, manejo, aptidão agrícola, entre outros (Oliveira et al., 1992). A classificação de solos é tema relevante no ensino e atuação profissional ligadas principalmente (embora não se restringindo) às áreas da Agropecuária, Geografia, Engenharia Civil e Geologia. Para que a classificação possa ser executada de acordo com critérios científicos foi organizado o Sistema Brasileiro de Classificação de Solos (SBCS).

No ensino de Ciência do Solo tem havido experiências variadas de "pedagogia ativa", que na maioria das vezes se auto-rotulam como experiências "construtivistas" (Eltz e Dalmolin, 1996; Braida, 1997). O ensino de solos não consiste apenas do tema da Classificação ou do domínio completo do SBCS. Por exemplo, nos cursos de agronomia esse tema se fragmenta e recombina em diferentes disciplinas relativas a Gênese, Levantamento, Classificação, Manejo, Física, Química e Fertilidade. Porém todos esses assuntos são inter-relacionados, interferem e/ou sofrem interferência da classificação e portanto se fazem presentes de alguma forma no SBCS.

Por outro lado, os materiais de referência do SBCS estão disponíveis em um ou mais volumes impressos, de manuseio trabalhoso, haja vista o processo não linear de busca das informações aplicadas na classificação e a linearidade de acesso do suporte impresso. Essa não correspondência entre as hierarquias de apresentação e de consulta às informações do SBCS torna a classificação uma tarefa enfadonha, especialmente para um não especialista. Esse aspecto se agrava porque existem várias centenas de classes de solos e surgem diversas relações complementares na interação entre as informações empregadas na definição de cada classe. Os relatos da observação empírica dos professores dessa área indicam que esses dois fatores - o acesso ineficiente às informações e o grande volume de classes distintas - dificultam a aprendizagem pois afetam negativamente a motivação dos alunos desde o início do processo.

Diante de tal quadro foi modelada e prototipada uma aplicação computacional para uso educacional que possibilite acesso eficiente às informações do SBCS. A aplicação tomou a forma de um hiperdocumento didático, ou seja, estruturado para capturar a semântica de uma abordagem didática. Esta abordagem consistiu da agregação em um hiperdocumento de um conjunto de informações do SBCS e seus principais relacionamentos hierárquicos, juntamente com um conjunto de diferentes representações multimídia de perfis de solos e seus processos de classificação, assim como das relações de pertença de cada perfil aos diferentes níveis categóricos. Os objetivos deste trabalho são os seguintes:

- Possibilitar a navegação rápida e estruturada abrangendo os principais temas do SBCS (classes de solos, critérios de classificação, horizontes e atributos diagnósticos) e exemplos de descrições de perfis de solo e sua classificação.

- Facilitar a abordagem didática do hiperdocumento SBCS no ensino de solos, através de uma modelagem e implementação que estimule seu uso por professores e alunos.

- Disponibilizar a descrição completa de pelo menos um perfil característico para cada uma das principais classes de solos de cada nível categórico.

- Incorporar recursos multimídia como uma alternativa para tornar mais interessante e estimulante a aprendizagem do SBCS, por exemplo, através de imagens estáticas de perfis exemplo e vídeos didáticos de seu processo de classificação associados as suas descrições textuais. 


\section{Domínio de aplicação: estrutura do SBCS}

O SBCS é uma chave taxionômica de classificação pedológica resultante de uma evolução do sistema americano, do qual herdou seus fundamentos. As modificações se iniciaram na década de 1950 e ainda hoje continua havendo uma dinâmica de revisões e aperfeiçoamentos, entretanto a publicação da versão oficial é responsabilidade da Empresa Brasileira de Pesquisa Agropecuária (EMBRAPA), baseando-se em projeto nacional de levantamento de solos coordenado pelo Centro de Pesquisa de Solos (Embrapa Solos). Este projeto se baseia no SBCS vigente (Embrapa, 1999).

O SBCS constitui-se de seis níveis categóricos, dos quais encontram-se efetivamente implementados os quatro primeiros: Ordens, Subordens, Grandes Grupos e Grupos. A identificação de um nível categórico inferior depende dos níveis superiores a que pertence. Por exemplo, o nome completo de uma Subordem inicia-se pelo nome da Ordem e assim sucessivamente para os demais níveis categóricos.

A classificação de um solo segundo o SBCS é feita partindo de uma amostragem a campo denominada Perfil (Lemos e Santos, 1996). Um perfil de solo é obtido pela abertura de uma trincheira ou corte perpendicular, de profundidade suficiente para deixar à mostra pelo menos seus horizontes A e B, ou seja, suas camadas superficial e sub-superficial, desde que ambas estejam presentes no local da amostragem (Figura 1).

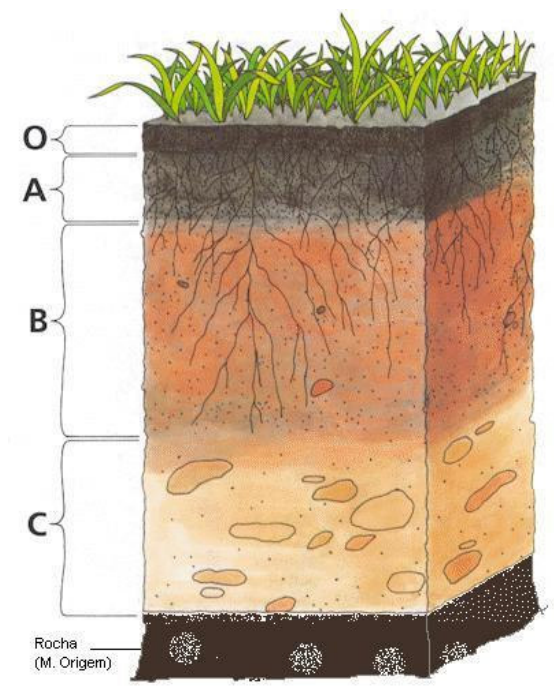

Figura 1 - Esquema de um perfil típico de solo e seus horizontes: O - orgânico; A - superficial; B - sub-superficial; C - intermediário; Rocha (material de origem).

No SBCS os critérios de classificação se compõem essencialmente de dois grupos de informações: a) horizontes diagnósticos: "camadas" características cuja presença/ausência e características morfológicas, físicas e químicas compõem os principais critérios de classificação quanto a Ordem e Subordem; b) atributos diagnósticos: características cuja presença/ausência e variações compõem os principais critérios de classificação do solo quanto a Grande Grupo e Grupo.

A descrição completa de um perfil de solo é composta de três blocos de informações: a) dados gerais: descritivos do contexto do perfil; b) descrição morfológica: para cada horizonte diagnóstico do perfil registra-se um conjunto de 
informações de avaliação visual e tátil, por exemplo, a "cor" segundo a carta de Munsell (1994); c) análises físicas e químicas: para cada horizonte diagnóstico presente no perfil é coletada uma amostra de solo para análise em laboratório. A tabela de resultados é relativamente extensa mas pode ser expressa por um conjunto de trincas do tipo: Horizonte Diagnóstico, Propriedade Física/Química, Valor Obtido. As informações resultantes das diversas análises visuais, táteis, físicas e químicas do perfil são submetidas comparativamente aos critérios do SBCS, fornecendo a classificação taxionômica do solo (Oliveira et al., 1992).

\section{$3 \quad$ Modelagem conceitual}

Nesta seção é apresentada a modelagem do hiperdocumento organizada para capturar a semântica de uma abordagem didática do SBCS. Foi adotada a técnica de modelagem HMT (Hypermedia Model Technique), proposta por Nemetz (1995), composta de quatro modelos: Objetos, Hipermídia, Navegação e Interface.

\subsection{Modelo de objetos}

O Modelo de Objetos do HMT segue a notação da OMT (Object Modelling Technique), conforme descrita por Rumbaugh et al. (1991), possibilitando descrever fundamentalmente as classes do domínio (grupamentos de objetos com propriedades similares), assim como os relacionamentos e os atributos dos objetos (Figura 2).

\subsection{Modelo de hipermídia}

O Modelo de Hipermídia é composto em duas etapas. Inicialmente são acrescentadas novas classes e relacionamentos necessários para prover os caminhos de acesso desejados e não capturados pelo Modelo de Objetos. Em seguida são identificadas e acrescentadas ao modelo as diferentes mídias que serão utilizadas.

\subsubsection{Acrescentando caminhos de acesso}

Ao Modelo de Hipermídia foram acrescentados caminhos de acesso direto entre Ordem e Grande Grupo, entre Ordem e Grupo e entre Subordem e Grupo através de três relacionamentos denominados "contém", necessários e não capturados inicialmente pelo Modelo de Objetos. Desta forma é possível, por exemplo, alcançar diretamente um Grande Grupo contido em uma Ordem sem ter que passar pela Subordem intermediária. O caminho inverso também é possível pois o relacionamento é bidirecional, ou seja, a partir de um Grande Grupo é possível alcançar diretamente a Ordem que o contém, sem ter que passar pela Subordem intermediária. A mesma regra vale para os demais caminhos adicionados.

A Tabela 1 resume os relacionamentos do Modelo de Hipermídia, assim como sua cardinalidade e direção de acesso. Por exemplo, uma Ordem apresenta um Horizonte Diagnóstico (cardinalidade "1 : 1") através de um relacionamento bidirecional, ou seja, pode-se alcançar o Horizonte Diagnóstico a partir da Ordem correspondente e vice-versa. Mas o relacionamento que determina que uma Ordem apresenta $\mathrm{N}$ atributos diagnósticos e que um Atributo Diagnóstico pode ser apresentado por $\mathrm{N}$ ordens (cardinalidade " $\mathrm{N}: \mathrm{N}$ ") está definido de maneira unidirecional, ou seja, a partir de uma Ordem pode-se alcançar todos os atributos diagnósticos da mesma, entretanto o inverso (de Atributo Diagnóstico para Ordem) não está previsto. O mesmo vale para os demais relacionamentos. 


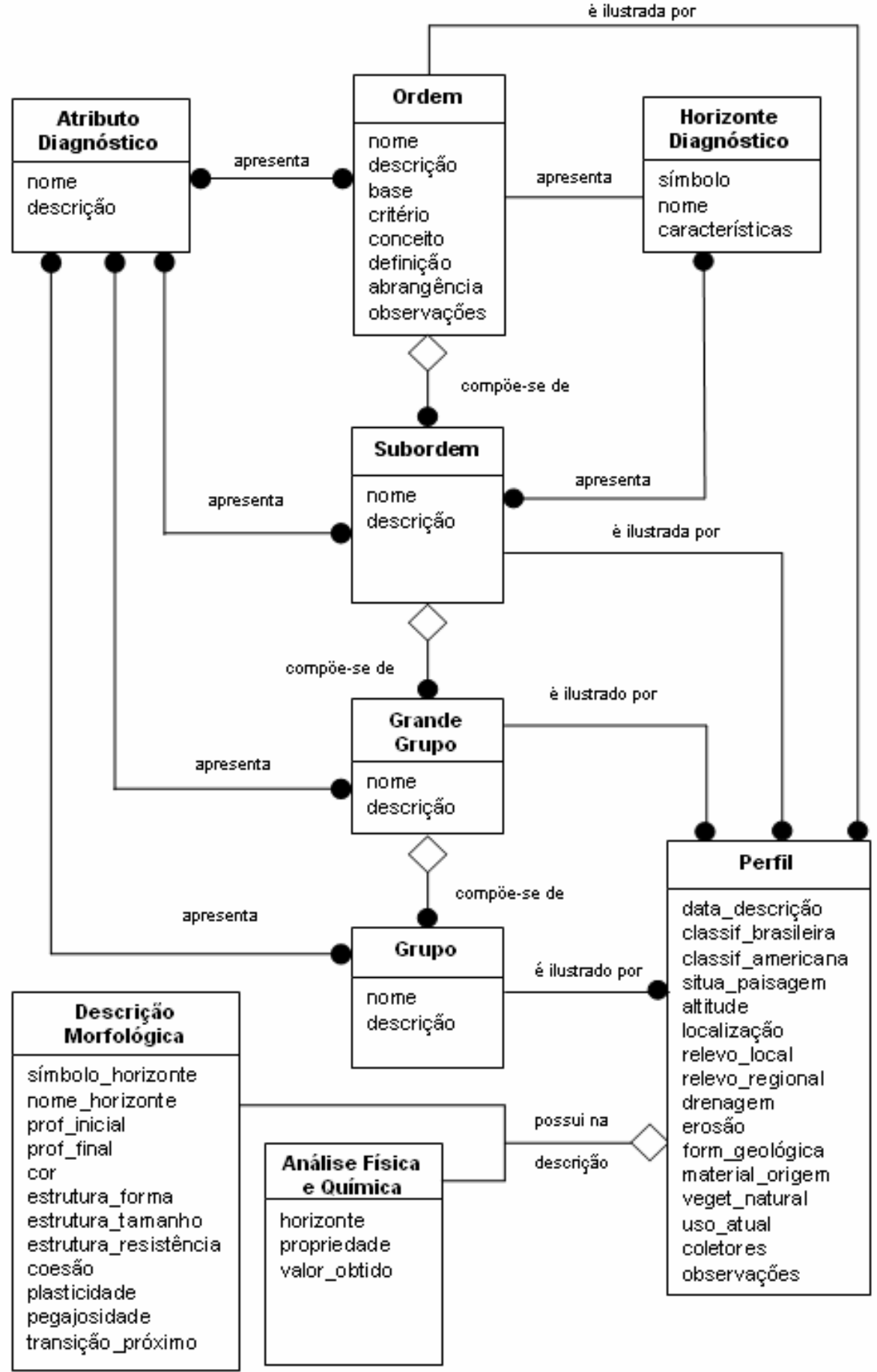

Figura 2 - Modelo de objetos e relacionamentos do hiperdocumento SBCS. 
Tabela 1 - Relacionamentos, cardinalidade e direção de acesso entre os objetos.

\begin{tabular}{|c|c|c|c|c|}
\hline Objeto & Relacionamento & Objeto & Cardinalidade & Direção \\
\hline Ordem & apresenta & Horizonte Diagnóstico & $1: 1$ & bidirecional \\
\hline Ordem & apresenta & Atributo Diagnóstico & $\mathrm{N}: \mathrm{N}$ & unidirecional \\
\hline Ordem & compõe-se de & Subordem & $1: \mathrm{N}$ & bidirecional \\
\hline Ordem & contém & Grande Grupo & $1: \mathrm{N}$ & bidirecional \\
\hline Ordem & contém & Grupo & $1: \mathrm{N}$ & bidirecional \\
\hline Ordem & é ilustrada por & Perfil & $1: \mathrm{N}$ & bidirecional \\
\hline Subordem & apresenta & Horizonte Diagnóstico & $\mathrm{N}: \mathrm{N}$ & unidirecional \\
\hline Subordem & apresenta & Atributo Diagnóstico & $\mathrm{N}: \mathrm{N}$ & unidirecional \\
\hline Subordem & compõe-se de & Grande Grupo & $1: \mathrm{N}$ & bidirecional \\
\hline Subordem & contém & Grupo & $1: \mathrm{N}$ & bidirecional \\
\hline Subordem & é ilustrada por & Perfil & $1: \mathrm{N}$ & bidirecional \\
\hline Grande Grupo & apresenta & Atributo Diagnóstico & $\mathrm{N}: \mathrm{N}$ & unidirecional \\
\hline Grande Grupo & compõe-se de & Grupo & $1: \mathrm{N}$ & bidirecional \\
\hline Grande Grupo & é ilustrado por & Perfil & $1: \mathrm{N}$ & bidirecional \\
\hline Grupo & apresenta & Atributo Diagnóstico & $\mathrm{N}: \mathrm{N}$ & unidirecional \\
\hline Grupo & é ilustrado por & Perfil & $1: \mathrm{N}$ & bidirecional \\
\hline Perfil & compõe-se de & Descrição Morfológica & $1: 1$ & bidirecional \\
\hline Perfil & compõe-se de & Análise Física e Química & $1: 1$ & bidirecional \\
\hline
\end{tabular}

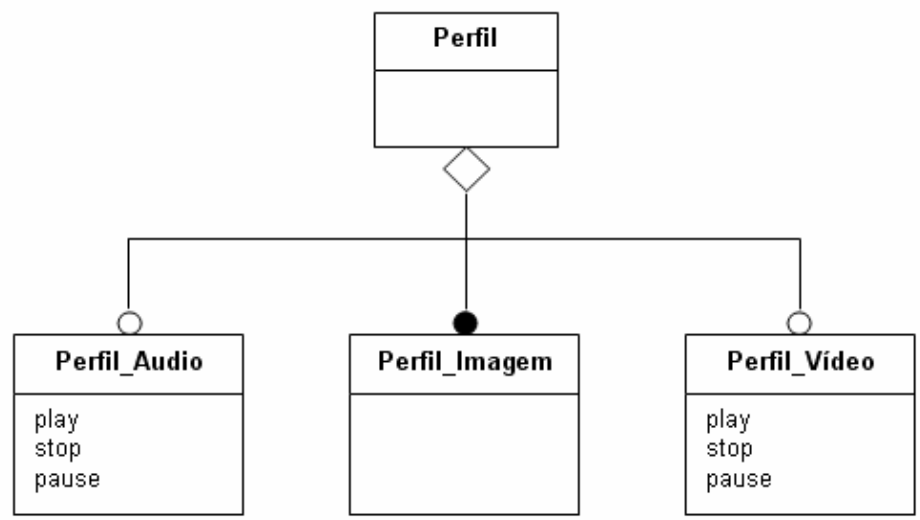

Figura 3 - Representação de um perfil de solo pela agregação de diferentes mídias. 6 


\subsubsection{Identificando as diferentes mídias}

No Modelo de Hipermídia quase todos os objetos são representados por um conteúdo em formato texto, com exceção dos objetos da classe Perfil (Figura 3) que, além de serem representados por uma agregação definida de Dados Gerais do Perfil + Descrição Morfológica + Análise Física e Química, podem ainda apresentar alternativamente a agregação de:

- uma descrição em vídeo didático, associado a texto e/ou imagens, do processo de análise e classificação do perfil, e/ou;

- uma ou mais imagens estáticas do perfil, e/ou;

- uma descrição em áudio didático, associado a texto e/ou imagens, do processo de análise e classificação do perfil.

\subsection{Modelo e contextos de navegação}

O Modelo de Navegação adotado é sensível aos contextos: Livre (todos os objetos de uma classe), Ordem, Subordem, Grande Grupo e Grupo (Figura 4). Durante a navegação, o contexto é mantido sempre que for possível e vantajoso para o usuário. Quando o usuário opta por "subir" na hierarquia do SBCS (direção Perfil $\rightarrow$ Grupo $\rightarrow$ Grande Grupo $\rightarrow$ Subordem $\rightarrow$ Ordem), o resultado é sempre "detalhar" o objeto que está acima. Por exemplo, a partir de uma Subordem qualquer no contexto "ALISSOLOS" (que é uma Ordem), a opção "ordem" (direção Subordem $\rightarrow$ Ordem) leva para detalhes do objeto "ALISSOLOS". Quando o usuário opta por "descer" na hierarquia do SBCS, o resultado é sempre "listar" os objetos que estão abaixo. Por exemplo, a partir dos detalhes do objeto "ALISSOLOS", a opção "grupo" leva para a lista dos grupos contidos na Ordem dos ALISSOLOS, que é mantida como contexto.

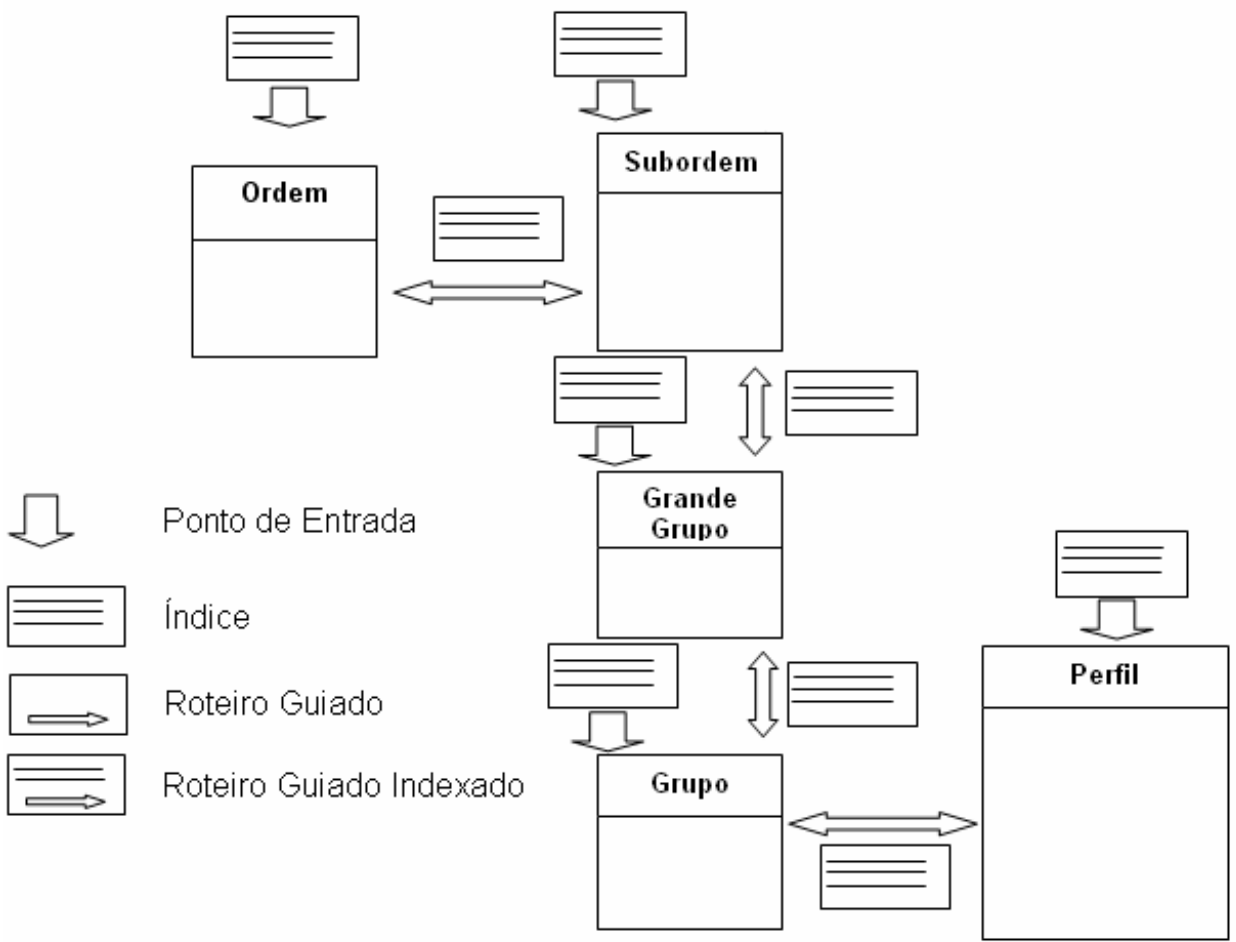

Figura 4 - Modelo de navegação: pontos de entrada, roteiros e índices. 


\subsection{Modelo de interface}

A interface da aplicação ${ }^{1}$ segue um padrão de páginas HTML com acesso via browser e elementos gráficos dos tipos: texto, hipertexto, imagens e multimídia incorporada (áudio e vídeo embedded). Os elementos representativos das ligações hipertextuais que permitem navegação ao usuário são do tipo texto (por exemplo, cada Ordem na lista da Figura 5 é a um hyperlink para o objeto correspondente). O Modelo de Interface se baseia em três frames (regiões) com a seguinte configuração básica: a) menu fixo: em disposição vertical, mais à esquerda, com as opções fixas de entrada ("Ordem, Subordem, Grande Grupo, Grupo, Perfil", Figuras 5 e 6); b) principal: mais abaixo e à direita, onde são apresentados os documentos da hiperbase, na forma de "lista" ou "detalhes" de objetos, inclusive os elementos multimídia (lista das ordens na Figura 5, "Observações" do objeto ALISSOLOS na Figura 6); c) cabeçalho: em disposição horizontal, mais acima e à direita, onde são apresentados, conforme o caso, o contexto de navegação ("ORDENS, Todas" na Figura 5, "ALISSOLOS" na Figura 6) e as opções de visualização do objeto apresentado ("Descrição | Base | Critério | Conceito | Definição | Abrangência | Observações" na Figura 6).

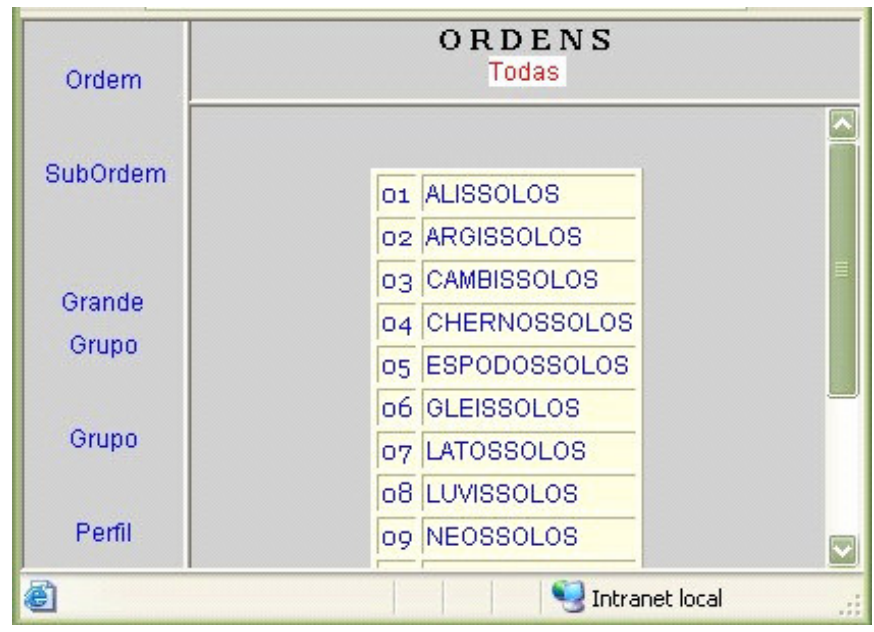

Figura 5 - Modelo de interface para o comportamento "listar" objetos de uma classe.

\begin{tabular}{|c|c|c|}
\hline \multirow[b]{3}{*}{ Subordem } & \multicolumn{2}{|c|}{$\begin{array}{c}\text { ALISSOLOS } \\
\text { | Descriçẫo | Base | Critério | Conceito | Definiçẫo | Abrangência | Observaçổes | }\end{array}$} \\
\hline & & 目 \\
\hline & Observações & \\
\hline $\begin{array}{l}\text { Grande } \\
\text { Grupo }\end{array}$ & $\begin{array}{l}\text { Alguns destes solos apresentam uma } \\
\text { acentuada diferenciação textural, sendo a } \\
\text { transição do A para o horizonte Bt clara ou } \\
\text { abrupta; em outros solos esta diferenciação } \\
\text { é menos pronunciada, e a transição do A } \\
\text { para o horizonte B textural ou B nítico é } \\
\text { normalmente clara, mais pelo contraste de } \\
\text { cor e estrutura, que pelo gradiente textural. } \\
\text { A seqüência de horizontes, tanto num caso } \\
\text { como no outro é A, Bt e C. }\end{array}$ & 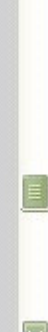 \\
\hline g & Intranet 1 & \\
\hline
\end{tabular}

Figura 6 - Modelo de interface para o comportamento "detalhar" um objeto. 


\section{$4 \quad$ Aspectos tecnológicos e de implementação}

Um protótipo do hiperdocumento foi implementado sobre um modelo de arquitetura cliente-servidor. O protótipo apresenta conteúdo na forma de páginas HTML estáticas e geradas dinamicamente, as quais incorporam informações de uma base de dados e conteúdo multimídia sincronizado no formato SMIL (Synchronized Multimedia Integration Language), incluindo áudio e vídeo sob demanda.

O protótipo foi disponibilizado na Internet ${ }^{2}$ baseando-se completamente em um conjunto de tecnologias e ferramentas de software de acesso gratuito. Do lado servidor foram empregadas as seguintes tecnologias: sistema operacional Linux, serviço HTTP baseado no Apache, interpretador da linguagem PHP para geração dinâmica de páginas HTML, gerenciador de banco de dados MySql, serviço de vídeo streaming baseado numa versão limitada do Helix ${ }^{\mathrm{TM}}$ Universal Server da Real Networks. Do lado cliente o usuário precisa ter instalado em sua máquina um navegador de Internet com suporte a CSS (Cascading Style Sheets) e o plugin Real Player ${ }^{3}$ com suporte a vídeo streaming e SMIL, para visualização do conteúdo multimídia.

$\mathrm{Na}$ construção do protótipo optou-se pela arquitetura cliente-servidor como uma forma de colocar a aplicação para acesso livre através da Internet e também pela disponibilidade de acesso e domínio da equipe sobre as ferramentas de desenvolvimento necessárias. Entretanto, a princípio o modelo conceitual apresentado pode ser materializado em outros formatos de implementação, por exemplo em CDROM educacional através de ferramentas de autoria de hipermídia.

A modelagem da base de dados do protótipo foi feita ad hoc e seria necessário refazê-la totalmente para uma versão cliente-servidor definitiva que priorize a robustez atendendo a critérios de engenharia de software. As classes e relacionamentos que dizem respeito a Atributos e Horizontes diagnósticos encontram-se modelados mas não estão implementados no protótipo atual.

\section{$5 \quad$ Considerações finais}

A aprendizagem do SBCS apresenta um potencial integrador pois o mesmo está relacionado intrinsecamente a toda Ciência do Solo. Os alunos poderiam tirar melhor proveito do SBCS para reforçar seus conhecimentos sobre os demais temas, ao aplicá-los na confrontação de critérios mobilizados pelo processo de classificação de solos. Entretanto, este potencial é desperdiçado pois o grande volume de informações envolvidas e seu acesso linear em manuais impressos, configuram-se numa dificuldade que leva os alunos a sentirem-se pouco motivados a aprender o SBCS aplicando-o.

Este trabalho apresentou a modelagem de um hiperdocumento educacional que possibilita a consulta hipertextual às informações do SBCS. Embora permita um acesso não linear aos conteúdos, a solução proposta captura a organização hierárquica do SBCS através de uma abstração do domínio em classes, objetos e relacionamentos.

O caráter didático do hiperdocumento é potencializado pela inclusão de descrições de perfis de solo (dados, imagens e tabelas de propriedades), associadas a representações (multimídia sincronizada com áudio/vídeo) do processo passo a passo de classificação destes mesmos perfis conforme executado por especialista. 
A aplicação pode ser utilizada como recurso educacional em cursos a distância ou presenciais, cabendo aos professores e alunos negociarem seu uso no ensino e aprendizagem de solos. Uma validação com alunos não foi possível até o momento. 


\section{Agradecimentos}

À Professora MSc Nilvania Aparecida de Mello, do Curso de Agronomia do CEFET-PR, doutoranda em Ciência do Solo (UFRGS), pelas discussões sobre ensino de solos, classificação dos perfis de solo do protótipo e consultoria técnica sobre o SBCS.

Ao Professor Doutor Luis Alberto Inda Junior, do Departamento de Solos da UFRGS, pela disponibilização de seu acervo particular de fotos de perfis de solo, anexadas a hiperbase do protótipo.

\section{Notas de texto}

${ }^{1}$ Os exemplos do Modelo de Interface se referem aos limites do protótipo atual.

${ }^{2}$ Protótipo disponível no endereço http://iate.ufrgs.br/ edilson/solos.

${ }^{3}$ Real Player e Helix Server disponíveis em: http://www.realnetworks.com.

\section{Referências Bibliográficas}

BRAIDA, J. A. (Ed.). A fragmentação do conhecimento e sua superação epistemológica. Documento final do III SIMPÓSIO BRASILEIRO SOBRE ENSINO DE SOLOS. Pato Branco, PR: Sociedade Brasileira de Ciência do Solo, CEFET-PR, 1997. 332p.

ELTZ, F. L. F.; DALMOLIN, R. S. D. (Eds.). A construção do conhecimento. Documento final do II SIMPÓSIO BRASILEIRO SOBRE ENSINO DE SOLOS. Santa Maria, RS: Sociedade Brasileira de Ciência do Solo, UFSM, 1996. 321p.

EMPRESA BRASILEIRA DE PESQUISA AGROPECUÁRIA. Sistema brasileiro de classificação de solos. Rio de Janeiro: EMBRAPA Solos, 1999.

LEMOS, R. C. de; SANTOS, R. D. Manual de descrição e coleta de solo no campo. $3^{a}$ ed. Campinas: Sociedade Brasileira de Ciência do Solo, 1996. 84p.

MUNSELL soil color charts. Rev. ed. New Windson: Kollmorgen Instruments, Macbeth Division, 1994. Não paginado.

NEMETZ, F. HMT modelagem e projeto de aplicações hipermídia. Porto Alegre: Instituto de Informática, CPGCC, UFRGS, 1995. Dissertação de Mestrado.

OLIVEIRA, J. B.; JACOMINE, P. K. T.; CAMARGO, M. N. Classes gerais de solos do Brasil: guia auxiliar para seu reconhecimento. $2^{\mathrm{a}}$ ed. Jaboticabal: FUNEP, 1992. $201 \mathrm{p}$.

RUMBAUGH, J. R.; BLAHA, M. R; LORENSEN, W.; EDDY, F.; PREMERLANI, W. Object-oriented modeling and design. New Jersey: Englewood Cliffs, 1991. 500p. 